\title{
BI-LEVEL PROGRAMMING MODEL AND ALGORITHMS FOR STOCHASTIC NETWORK WITH ELASTIC DEMAND
}

\author{
Xiang Zhang, Hao Wang, Wei Wang \\ School of Transportation, Southeast University, China
}

Submitted 25 February 2013; resubmitted 21 April 2013; accepted 20 August 2013

\begin{abstract}
Based on a state-of-the-art review of the Road Network Design Problem (RNDP), this paper proposes a bi-level programming model for the RNDP as well as algorithms for it. In the lower level of the proposed model, the elastic-demand Stochastic User Equilibrium (SUE) model is adopted to coincide well with characteristics of users behavior, and additionally, the parameter calibration method for the model is developed based on the Logit path choice model. In the upper level of the proposed model, the consumer surplus is maximized to improve the social benefit of a network in consideration of the travel demand, the construction cost, the off-gas emissions and the saturation level. The algorithm for the lower-level model is developed based on the descent iteration method, Dijkstra's algorithm and linear search technology. A modified Genetic Algorithm (GA) is developed as the algorithm for the whole bi-level model, which takes designed elitist selection operator, adaptive cross operator, mutation operator and niche technology into consideration. The proposed model and algorithms are applied to a numerical example. The results demonstrate the validity and efficiency of the model and algorithms, which shows a bright prospect of the application in RNDP.
\end{abstract}

Keywords: road network; traffic design; bi-level program; elastic-demand stochastic user equilibrium; genetic algorithm.

\section{Introduction}

Road Network Design Problem (RNDP) is regarded as one of the most complicated and challenging problems in transportation field. RNDP consists in optimizing a network to maximizing social benefit, by means of adding new links and improving existing links, with respect to a set of factors (traffic, cost, environment, etc.) (Cascetta 2001). Given an existing urban network, the problem is to define the optimal configuration of the road network in terms of link attributes (grade, capacity, etc.). In RNDP, both users and non-users (system planners in charge of traffic, investment, environment, etc.) participate in the decision process, who are interrelated and interactional, so RNDP is a typical bi-level program (Chiou 2009). As a more comprehensive review, Yang and Bell (1998) presented a review analysis of models and algorithms for RNDP. In recent years, various advanced bi-level programming models and algorithms for RNDP have been proposed (Luathep et al. 2011; Wang, Lo 2010; Bagloee, Ceder 2011).

For most bi-level programming models, the lower level is represented as a User Equilibrium (UE) assignment problem, while the upper level can be regarded as an investment decision problem. In the lower level, UE assignment problem can be classified as Deterministic User Equilibrium (DUE) and Stochastic User Equilibrium (SUE). Also, two types of travel demands are usually concerned with UE problems, namely, the fixed demand and the elastic demand. Thus, there are several kinds of assignment models, in which, the fixed-demand DUE model is adopted under most circumstances. Asakura and Sasaki (1990) used elastic-demand DUE model in RNDP in 1990 for the first time; Patriksson (2008) made a pioneer study in which the fixed-demand SUE model is adopted. Admittedly, the elastic-demand SUE model corresponds with users' behavior the best (Chen, Alfa 1991), but it can hardly be used in RNDP due to its complexity. In the upper level, most traditional models emphasized minimizing system costs, including travel time and/or construction cost, as an ultimate objective. As an exception, Friesz et al. (1993) established a multi-factors RNDP model, taking both system costs and vehicle miles travelled into consideration for the first time. In recent years, resource and environment problems have increased seriously, so that some other factors, including the use ratio of link and the off-gas emissions, are thought highly of by researchers (Sharma et al. 2009;

Corresponding author: Hao Wang

E-mail: haowang@seu.edu.cn 
Qin et al. 2010; Chen et al. 2010). Multi-factors programs are becoming the new hotspot in RNDP.

So far, previous algorithms for elastic-demand SUE model have great limitations in use, in which, for instance, method of successive average only adapts well to rail transit network and public transit network, while method of network diagram transformation produces fictitious links inevitably (Zhou, J. 2001; Zhou, Z. 2008). For bi-level programs, many heuristic algorithms are used in previous researches, including simulated annealing algorithm (Friesz et al. 1992), neural network algorithm (Xiong, Schneider 1995), Genetic Algorithm (GA) (Drezner, Wesolowsky 2003), ant system algorithm (Poorzahedy, Abulghasemi 2005), hybrid meta-heuristic algorithm (Poorzahedy, Rouhani 2007), particle swarm optimization (He et al. 2013), among which GA is most widely used because of its parallelism and compatibility (Cantarella, Vitetta 2006). Drezner, Salhi (2002) and Karoonsoontawong, Waller (2006) compared performance of these advanced heuristics in test problems, respectively. Both studies demonstrate that GA is superior in finding optimal solutions of RNDP. Despite this, GA also has shortcomings, such as slow convergence rate, premature convergence. Therefore, improvement measures should be taken.

In this paper, a bi-level programming model for RNDP is presented. The model adopts elastic-demand SUE model in the lower level to describe users' behavior in networks. Meanwhile, a new parameter calibration method for this model is provided, which is developed by minimizing the difference between theoretical traffic demands and actual ones. The upper-level subprogram takes multi-factors into consideration, including the travel demand, the construction cost, the off-gas emissions and the saturation level. Additionally, solution algorithms are also developed for both elastic-demand SUE model and proposed bi-level programming model, based on some classic methods including descent iteration (Ortega, Rheinboldt 1970), Dijkstra's algorithm (Kung et al. 1984), linear search technology (Florian 1977), GA and so on.

In Section 1, a bi-level programming model for RNDP is proposed. In Section 2, solution algorithms are developed for the proposed model. Section 3 presents a numerical example for a specific network. Finally, some conclusions and future work are summarized in last section.

\section{Bi-Level Programming Model}

\subsection{Notations}

Notations used throughout the paper are listed as follows unless otherwise are specified:

$A$ - set of links in a network, including probably added links;

$a$ - link index, where $a \in A$;

$C_{a}$ - capacity of link $a$;

$D_{i j}(\cdot)$ - travel demand function of O-D pair $i j$;

$f_{a}-$ flow for link $a$;

$f$ - link flow vectors whose elements are $f_{a}$; $h_{k}^{i j}$ - flow for path $k$ of O-D pair $i j$;

$h$ - path flow vector whose elements are $h_{k}{ }^{i j}$;

$i$ - origin node index;

$i j$ - origin-destination $(\mathrm{O}-\mathrm{D})$ pair index;

$j$ - destination node index;

$K$ - set of paths;

$K_{i j}$ - set of paths connecting origin $i$ with destination $j$;

$k$ - path index, where $k \in K$;

$L_{a}$ - length of link $a$;

$q_{i j}$ - travel demand between O-D pair $i j$;

$q-N \times 1$ vector of $\mathrm{O}-\mathrm{D}$ travel demands whose elements are $q_{i j}$;

$t_{a}-$ travel cost on link $a$;

$t_{k}^{i j}$ - travel cost on path $k$ of O-D pair $i j$;

$Z_{1}, Z_{2}$ - objective function values of upper-level subprogram and lower-level subprogram, respectively;

$\theta$ - accuracy degree of perceived travel cost;

$\delta_{a, k}$ - link-path incidence coefficient, whose value is equal to one if link $a$ belongs to path $k$, zero otherwise.

\subsection{Lower-Level Subprogram}

In the bi-level programming model for RNDP, the lower level adopts UE assignment model. As it is known to us, a famous phenomenon called Braess's Paradox exists in RNDP, which indicates that if path choice behavior of users is not taken into comprehensive consideration in RNDP, the running statement cannot be improved and what is more, crowdedness degree might ascend in a network (Braess 1968). For this reason, the assignment model in the lower level must coincide well with characteristics of users behavior. There are two main characteristics, namely, the uncertainties of both travel demand and path choice. Uncertainty of travel demand indicates that travel demand is elastic which varies with network reformation, while uncertainty of path choice indicates that users are not always choosing the very path with minimum cost due to limitation of their knowledge level and information obtainment. To correspond with these two characteristics, model for SUE assignment with elastic demand is adopted in the lower-level subprogram in this research. The elastic-demand SUE model can be formulated as a mathematical program as follows (Zhou, Xu 2001):

$$
\begin{aligned}
& \min _{(f, q, h)} Z_{2}=\sum_{a} \int_{0}^{f_{a}} t_{a}(x) d x+\frac{1}{\theta} \sum_{i j} \sum_{k} h_{k}^{i j} \cdot\left(\ln h_{k}^{i j}-1\right)- \\
& \frac{1}{\theta} \sum_{i j} q_{i j} \cdot\left(\ln q_{i j}-1\right)-\sum_{i j} \int_{0}^{q_{i j}} D_{i j}^{-1}(x) d x
\end{aligned}
$$

subject to:

$$
\begin{aligned}
& h_{k}^{i j} \geq 0, \forall i, j, k ; \\
& \sum_{k} h_{k}^{i j}=q_{i j}, \forall i, j ; \\
& f_{a}=\sum_{i} \sum_{j} \sum_{k} h_{k}^{i j} \delta_{a, k}, \forall a ;
\end{aligned}
$$

where: constraints $(1 b-d)$ are flow constraints for UE 
assignment models. Specifically, constraint (1b) denotes the non-negativity on path flows; constraint (1c) is the travel demand conservation constraint and constraint (1d) is a definitional constraint that sums up all path flows passing through a given link. In the formulation above, $t_{a}$ can be expressed as travel time on link $a$, which is calculated by Bureau of Public Road function (Highway Capacity Manual 2010):

$$
t_{a}=t_{a 0} \cdot\left(1+0.15 \cdot\left(\frac{f_{a}}{C_{a}}\right)^{4}\right),
$$

where: $t_{a 0}$ is free-flow travel time which equals to the link length divided by the free-flow speed.

Though objective function (1a) has no real meanings by itself, it represents SUE Principle that no traveler can improve his or her travel cost that he or she perceives by unilaterally changing routes. The route choice process in this case is probabilistic rather than deterministic. A distinction exists between the actual travel cost and perceived travel cost (Zhou, Xu 2001):

$$
T_{k}^{i j}=t_{k}^{i j}+\varepsilon_{k}^{i j},
$$

where: $T_{k}^{i j}$ is the perceived travel cost on path $k$ of O-D pair $i j, \varepsilon_{k}^{i j}$ is a random variable whose expectation is equal to zero.

$\varepsilon_{k}^{i j}$ can be expressed in the following form (Zhou, $\mathrm{Xu}$ 2001):

$$
\varepsilon_{k}^{i j}=\frac{1}{\theta} \cdot \xi_{k}^{i j},
$$

where: $\theta$ is a constant that represents accuracy degree of perceived travel cost. The higher its value is, the more probability there is for users to choose the path with minimum travel cost. $\xi_{k}{ }^{i j}$ is a kind of independently and identically distributed random variates. The corresponding path choice model is Logit model, which is equivalent to elastic-demand SUE model and provides a closed-form expression of path choice probabilities (Kuang et al. 2007):

$$
P_{k}^{i j}=\frac{\exp \left(-\theta \cdot t_{k}^{i j}\right)}{\sum_{w \in K_{i j}} \exp \left(-\theta \cdot t_{w}^{i j}\right)} .
$$

Then flow for path $k$ can be expressed as follows:

$$
h_{k}^{i j}=q_{i j} \cdot P_{k}^{i j} \text {. }
$$

In model (1), travel demand is elastic and has direct relationship with travel cost, which can be calculated as follows (Kuang et al. 2007):

$$
q_{i j}=D_{i j}\left(S_{i j}\right)=q_{i j}^{0} \cdot \exp \left(-\beta \cdot S_{i j}\right),
$$

where: $q_{i j}^{0}$ is the maximum potential travel demand between O-D pair ij. There are many factors that influence $q_{i j}^{0}$ including population, income, employment level, vehicle stock at origin node and stopping attraction at destination node. Values of $q_{i j}^{0}$ are determined by resident travel survey and travel demand prediction. $\beta$ is a constant that represents the sensitivity of travel demand to its cost. Generally speaking, the higher network crowdedness degree is, the larger the value of $\beta$ is. $S_{i j}$ is expectation of minimum perceived travel cost, which can be calculated as follows (Kuang et al. 2007):

$$
S_{i j}=-\frac{1}{\theta} \cdot \ln \left(\sum_{k \in K_{i j}} \exp \left(-\theta \cdot t_{k}^{i j}\right)\right) .
$$

Combining equations (7) and (8), $q_{i j}$ can be expressed as follows:

$$
\begin{aligned}
& q_{i j}=q_{i j}^{0} \cdot \exp \left(-\frac{\beta}{\theta} \cdot \ln \left(\sum_{k \in K_{i j}} \exp \left(-\theta \cdot t_{k}^{i j}\right)\right)\right)= \\
& q_{i j}^{0} \cdot\left(\sum_{k \in K_{i j}} \exp \left(-\theta \cdot t_{k}^{i j}\right)\right)^{-\beta / \theta} .
\end{aligned}
$$

Combining equations (5), (6) and (9), $h_{k}^{i j}$ can be expressed as follows:

$$
\begin{aligned}
& h_{k}^{i j}=D\left(S_{i j}\right) \cdot P_{k}^{i j}=q_{i j}^{0} \cdot\left(\sum_{w \in K_{i j}} \exp \left(-\theta \cdot t_{w}^{i j}\right)\right)^{-\beta / \theta} \times \\
& \frac{\exp \left(-\theta \cdot t_{k}^{i j}\right)}{\sum_{w \in K_{i j}} \exp \left(-\theta \cdot t_{w}^{i j}\right)}=q_{i j}^{0} \cdot\left(\sum_{w \in K_{i j}} \exp \left(-\theta \cdot t_{w}^{i j}\right)\right)^{-\beta / \theta-1} \times \\
& \exp \left(-\theta \cdot t_{k}^{i j}\right) .
\end{aligned}
$$
follows:

In equations (9) and (10), $t_{k}^{i j}$ can be calculated as

$$
t_{k}^{i j}=\sum_{a} t_{a} \cdot \delta_{a, k},
$$

where $t_{a}$ has direct connection with flows. Hence, equations (9) and (10) are implicit functions of flow solutions in model (1), so flow solutions can not be obtained only with these two equations.

Admittedly, it is almost impossible for any model to avoid Braess's Paradox absolutely, including elasticdemand SUE model. For instance, as $\theta$ expands to infinity, SUE solutions approach DUE solutions, and thus Braess's Paradox can be reproduced to an arbitrary degree. The extent to which elastic-demand SUE model is consistent with users' behavior depends on precision degree of values of $\beta$ and $\theta$. However, in previous studies, values of $\beta$ and $\theta$ are not quantitatively determined, but empirically estimated according to crowdedness degree of a network, knowledge level and information obtainment of users. According to previous literature, reference range of $\theta$ is between 0.1 and 3.5 (Zhou, $\mathrm{Xu} 2001$ ); reference range of $\beta$ is between 0.01 and 0.1 (unit: $p c u / h$ for demand and $\min$ for cost) (Li et al. 2001). Actually, either of equations (9) and (10) can help determine parameters $\beta$ and $\theta$ quantitatively. In this paper, equation (9) is used to develop a parameter calibration method 
for the elastic-demand SUE model. The main steps of the procedure as depicted in Fig. 1 are summarized:

\section{Step 1. Initialization:}

Set initial solutions, $\beta=0.01$ and $\theta=0.1$.

Step 2. Gaining theoretical value of travel demand:

- Calculate travel cost on each path by equations (2) and (11).

- Calculate $q_{i j}$ with equation (9), which serves as theoretical value of travel demand between each O-D pair.

Step 3. Termination:

- Besides theoretical value $q_{i j}$, there is also actual travel demand $q_{i j}^{\prime}$, which represents travel demand between $\mathrm{O}-\mathrm{D}$ pair $i j$ on the existing road network and can be got through traffic investigations. Calculate the error $\varepsilon_{1}$ between $q_{i j}$ and $q_{i j}^{\prime}$ as follows:

$$
\varepsilon_{1}=\frac{\sqrt{\sum_{i j}\left(q_{i j}-q_{i j}^{\prime}\right)^{2}}}{\sum_{i j} q_{i j}^{\prime}} .
$$

- If $\varepsilon_{1}<\varepsilon_{0}$ (where: $\varepsilon_{0}$ is the maximum permissible error), output current values of $\beta$ and $\theta$ as final results and stop the whole solving procedure. Otherwise, continue this step.

- If $\theta=3.5$, then reset $\beta$ and $\theta, \beta=\beta+0.01, \theta=$ 0.1 . Otherwise, reset $\theta, \theta=\theta+0.1$.

Go to Step 2.

Remark 1. The method above uses flows in an original network to determine parameters with implicit function (9) of flow solutions for programming model (1). The aim is to search the optimal values of $\beta$ and $\theta$ which can make theoretical travel demands gained by Logit model correspond well with actual flows under current circumstance.

Remark 2. The whole procedure amounts to a double-deck circulation structure. In outer circulation, the feasible solution of $\beta$ ranges from 0.01 to 0.1 with the interval of 0.01 , while in intimal circulation, the feasible

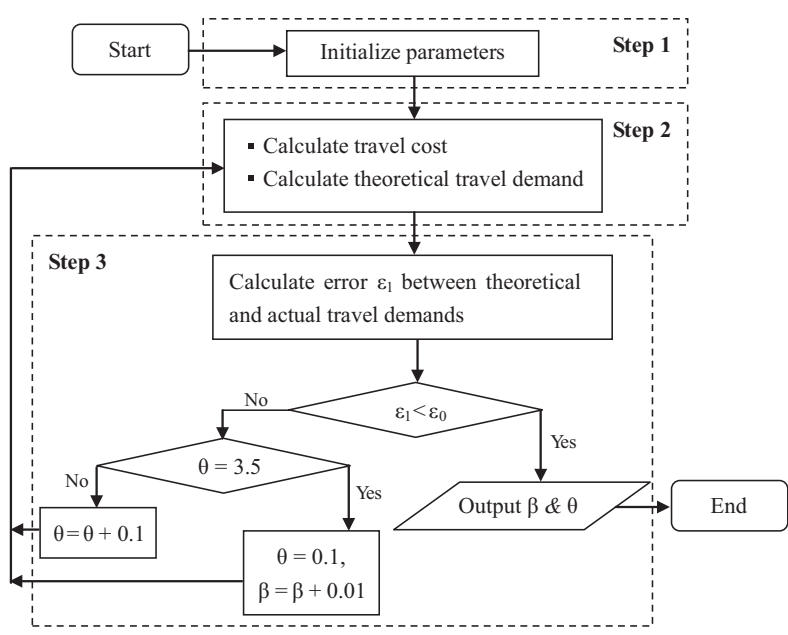

Fig. 1. Parameter calibration for elastic-demand SUE model solution of $\theta$ ranges from 0.1 to 3.5 with the interval of 0.1 . The optimization starts from $\beta=0.01$ and $\theta=0.1$, and continues until the error in Step 3 is less than the maximum permissible one.

\subsection{Upper-Level Subprogram}

In bi-level programming models for RNDP, the upper level is usually represented as some investment decision problem. In most construction projects, measures of both adding new links and improving existing links are taken, and the corresponding problem is a mixed RNDP. Hence, mixed RNDP model is adopted in the upper level. In consideration of elastic travel demand in the lower level, the upper level should not pursue the minimum of construction costs or travel costs. Otherwise, travel demands would drop markedly, which is contrary to the original intention of network design. Under elastic demand, the upper-level subprogram should improve social benefit by maximizing consumer surplus, which accounts for the difference between total user benefit and total social cost. In RNDP, consumer surplus is expressed by the difference between travel demand and construction cost in common circumstances, while in this paper, off-gas emissions is also taken into consideration. With the goal of maximizing consumer surplus, it is expected to attract more travel demands at the expense of fewer construction costs as well as fewer pollutant emissions. Also, saturation level is taken into account to guarantee appropriate use ratio and service level on links. Thus, the upper-level subprogram can be formulated as a nonlinear integer program as follows:

$$
\begin{aligned}
& \max _{Y} Z_{1}=\sum_{i j} q_{i j}(\mathbf{Y})-\theta_{1} \cdot \sum_{a} G_{a}\left(y_{a}\right)- \\
& \theta_{2} \cdot \sum_{a} h_{a} \cdot L_{a} \cdot f_{a}(\mathbf{Y})
\end{aligned}
$$

subject to:

$$
\begin{aligned}
& y_{a} \in\{0,1,2, \ldots, m\} \quad \forall a \in A ; \\
& R_{l} \leq R_{a} \leq R_{h} \quad \forall a \in A,
\end{aligned}
$$

where: $\mathrm{Y}$ is design vector of the program, which involves a set of integer elements $y_{a}$ that represent attributes of link $a$ after network reformation. $q_{i j}(\mathbf{Y})$ and $f_{a}(\mathbf{Y})$ are travel demands under certain road network reformation scheme which can be obtained by solving lower-level subprogram; $G_{a}\left(y_{a}\right)$ is the construction-cost function of link $a ; R_{a}$ is the saturation level on link $a ; R_{l}$ and $R_{h}$ are the upper and lower bounds of saturation level respectively; $\theta_{1}$ is a constant coefficient matching units of travel demand and construction cost; $\theta_{2}$ is a constant coefficient matching units of travel demand and off-gas emissions; $h_{a}$ is off-gas emissions coefficient of link $a$ which represents off-gas emissions of an equivalent car when it runs normally for a single-unit distance on link $a$.

Remark 3. According to professional standard (Ministry of Housing and... 1991; Highway Capacity Manual 2010) and reference (Guo 2009), link grades and attributes for urban areas are summarized in Table 1. In construction projects, attribute values can be changed flexibly in accordance with actual situation. 
Table 1. Values of $y_{a}$ and the corresponding link attributes

\begin{tabular}{lcccccc}
\hline$y_{a}$ & Link grade & Link category & Lane number & $\begin{array}{c}\text { Design speed } \\
{[\mathrm{km} / \mathrm{h}]}\end{array}$ & $\begin{array}{c}\text { One-way capacity } \\
{[\mathrm{pcu} / \mathrm{h}]}\end{array}$ & $\begin{array}{c}\text { Cost } \\
{[1000 \text { yuan } / \mathrm{km}]}\end{array}$ \\
\hline 1 & I & express way & 8 & $60-80$ & 4800 & 1600 \\
\hline 2 & II & express way & 8 & $60-80$ & 3600 & 1300 \\
\hline 3 & III & arterial road & 6 & $30-60$ & 4000 & 1000 \\
\hline 4 & IV & arterial road & 6 & $30-60$ & 3000 & 800 \\
\hline 5 & V & sub-arterial road & 4 & $20-50$ & 2400 & 600 \\
\hline 6 & VI & sub-arterial road & 4 & $20-50$ & 1600 & 400 \\
\hline 7 & VII & branch & 2 & $20-40$ & 1500 & 300 \\
\hline 8 & VIII & branch & 2 & $20-40$ & 750 & 200 \\
\hline 0 & Not add or improve link & & & & \\
\hline
\end{tabular}

Notes: 1) for existing links, construction cost equals the expense on link performance improvements; 2) 1 yuan $\approx 0.16$ dollar.

Remark 4. In RNDP, there is a phenomenon called Pollutant Emissions Paradox, which indicates that volume of off-gas emissions rises dramatically though measures of adding new links or improving existing links in network design (Zhao et al. 2005). In order to avoid decision-making failure, off-gas emissions should be taken into consideration as shown in objective function (12a), where off-gas emissions coefficient can be expressed as a polynomial function with respect to travel speed (Anderson et al. 1996):

$$
h_{a}=b_{0}+b_{1} \cdot v_{a}+b_{2} \cdot v_{a}{ }^{2}+b_{3} \cdot v_{a}{ }^{3},
$$

where: $v_{a}$ is the mean speed on link $a ; b_{1}, b_{2}, b_{3}, b_{4}$ are constant coefficients which are necessary to determine according to specific areas with the help of curve fitting calculations (Wang 2002).

Remark 5. In terms of social benefits, $\theta_{1}$ indicates that incremental social benefits caused by decreasing one-unit construction cost amount to those caused by increasing $\theta_{1}$-unit travel demands, while $\theta_{2}$ indicates that incremental social benefits caused by decreasing one-unit off-gas emissions amount to those caused by increasing $\theta_{2}$-unit travel demands. Thus, these two coefficients essentially reflect the importance of traffic benefit and social benefits. For a construction project, if economical efficiency is relatively more important, then the upper bound of construction cost is lower and the minimum expected travel demand contributed by oneunit construction cost is more. Therefore, the value of $\theta_{1}$ needs to be set higher in this case. Similarly, if environmental efficiency is of more importance, value of $\theta_{2}$ needs to be set higher, while if traffic benefit is of more importance, values of both $\theta_{1}$ and $\theta_{2}$ should be set lower. In fact, the upper-level formulation can be expressed in another form:

$$
\max _{Y} Z_{1}=\sum_{i j} q_{i j}(\mathbf{Y})
$$

subject to:

$$
\begin{aligned}
& \sum_{a} G_{a}\left(y_{a}\right) \leq A_{1} ; \\
& \sum_{a} h_{a} \cdot L_{a} \cdot f_{a}(\mathbf{Y}) \leq A_{2} ;
\end{aligned}
$$

$$
\begin{aligned}
& y_{a} \in\{0,1,2, \ldots, m\}, \forall a \in A ; \\
& R_{l} \leq R_{a} \leq R_{h}, \forall a \in A .
\end{aligned}
$$

It can be proved in mathematics that formulation (14) is identical with formulation (12) if Lagrange multipliers of constraint (14b) and (14c) are equal to $\theta_{1}$ and $\theta_{2}$ respectively. In this case, negative correlation exists between $\theta_{1}$ and $A_{1}$, as well as $\theta_{2}$ and $A_{2}$, which corresponds to the analysis above.

\subsection{Bi-Level Program}

Based on Sections 1.2 and 1.3, the bi-level programming model for mixed RNDP can be established as follows:

$$
\begin{aligned}
& \max _{Y} Z_{1}=\sum_{i j} q_{i j}(\mathrm{Y})-\theta_{1} \cdot \sum_{a} G_{a}\left(y_{a}\right)- \\
& \theta_{2} \cdot \sum_{a} h_{a} \cdot L_{a} \cdot f_{a}(\mathbf{Y})
\end{aligned}
$$

subject to:

$$
\begin{aligned}
& y_{a} \in\{0,1,2, \ldots, m\}, \forall a \in A ; \\
& R_{l} \leq R_{a} \leq R_{h}, \forall a \in A,
\end{aligned}
$$

where the flow set can be obtained by solving the lowerlevel model below:

$$
\begin{aligned}
& \min _{(f, q, h)} Z_{2}=\sum_{a} \int_{0}^{f_{a}} t_{a}(x) d x+\frac{1}{\theta} \cdot \sum_{i j} \sum_{k} h_{k}^{i j} \times \\
& \left(\ln h_{k}^{i j}-1\right)-\frac{1}{\theta} \sum_{i j} q_{i j} \cdot\left(\ln q_{i j}-1\right)- \\
& \sum_{i j} \int_{0}^{q_{i j}} D_{i j}^{-1}(x) d x
\end{aligned}
$$

subject to:

$$
\begin{aligned}
& h_{k}^{i j} \geq 0, \forall i, j, k ; \\
& \sum_{k} h_{k}^{i j}=q_{i j}, \forall i, j ; \\
& f_{a}=\sum_{i} \sum_{j} \sum_{k} h_{k}^{i j} \cdot \delta_{a, k}, \forall a .
\end{aligned}
$$

Compared with some previous advanced RNDP models (Luathep et al. 2011; Farvaresh, Sepehri 2011; 
Chen et al. 2011), this model has advantages mainly in following aspects:

- For the lower-level subprogram:

- in consideration of two main characteristics in assignment (uncertainties of the travel demand and the path choice), the elastic-demand SUE model is adopted to coincide well with characteristics of users' path choice behaviour;

- values of coefficients can be quantitatively determined by implicit functions of flow solutions for the model.

- For the upper-level subprogram: objective in the upper level is maximizing consumer surplus to pursue social benefits by measures of both adding new links and improving existing links, taking multifactors including travel demand, construction cost, off-gas emissions and saturation level into consideration. What's more, the importance of traffic benefit, economical efficiency and environmental efficiency in construction projects can be represented through adjusting unit-matching coefficients.

\section{Solution Algorithms}

\subsection{Algorithm for Lower-Level Subprogram}

Nowadays, only a few algorithms have been proposed to solve elastic-demand SUE model, and existing algorithms are more or less defective. In this research, a utility algorithm for elastic-demand SUE model is developed based on some classic methods such as descent iteration method, Dijkstra's algorithm and linear search technology. Main steps of solution procedure as depicted in Fig. 2 are summarized:

Step 1. Initialization:

- Set initial iteration counter $n=0$.

- Set initial solution set $\left\{f_{a}^{1}\right\},\left\{q_{i j}^{1}\right\}$ and $\left\{h_{k}^{i j, 1}\right\}$, which meet flow constraints for UE assignment models.

Step 2. Determining iteration direction:

- Calculate travel cost on each link $a$ by equation (2).

- With the help of Dijkstra's algorithm, find out the very path $m$ with minimum travel cost $t_{m}^{i j, n}$ of each O-D pair $i j$ and calculate $\mu_{i j}^{i 0}$ representing minimum travel cost of each $\mathrm{O}-\mathrm{D}$ pair $i j$ when there is none flow in the whole network.

- Calculate $D_{i j}^{-1}\left(q_{i j}^{n}\right)$ by equation (5). Calculate augmentation flow for each path $g_{m}^{i j, n}$ as follows:

if $t_{m}^{i j, n}<D_{i j}^{-1}\left(q_{i j}^{n}\right)$, then $g_{m}^{i j, n}=D_{i j}\left(\mu_{i j}^{0}\right)$

and $g_{k}^{i j, n}=0, \forall k \neq m$;

if $t_{m}^{i j, n}>D_{i j}^{-1}\left(q_{i j}^{n}\right)$, then $g_{k}^{i j, n}=0, \forall k$;

if $t_{m}^{i j, n}=D_{i j}^{-1}\left(q_{i j}^{n}\right)$, then $g_{m}^{i j, n}=\bar{q}_{i j} / 2$

and $g_{k}^{i j, n}=0, \forall k \neq m$.

- Based on $g_{m}^{i j, n}$, calculate augmentation flow for each link $y_{a}^{n}$ and augmentation travel demand between each O-D pair $v_{i j}^{n}$ as follows:

$$
\begin{aligned}
& y_{a}^{n}=\sum_{i} \sum_{j} \sum_{k} g_{k}^{i j, n} \cdot \delta_{a, k}^{i j}, \forall a ; \\
& v_{i j}^{n}=\sum_{k} g_{k}^{i j, n}, v \forall i, j .
\end{aligned}
$$

Step 3. Determining iteration step size:

Gain iteration step size by solving the following one-dimensional program with the help of linear search technology, specifically Fibonacci method (Bergum et al. 1992).

$$
\begin{aligned}
& \min Z\left(\alpha^{n}\right)=\sum_{a} \int_{0}^{f_{a}^{n}+\alpha^{n} \cdot\left(y_{a}^{n}-f_{a}^{n}\right)} t_{a}(x) d x+ \\
& \frac{1}{\theta} \sum_{i j} \sum_{k}\left(h_{k}^{i j, n}+\alpha^{n} \cdot\left(g_{k}^{i j, n}-h_{k}^{i j, n}\right)\right) \times \\
& \left(\ln \left(h_{k}^{i j, n}+\alpha^{n} \cdot\left(g_{k}^{i j, n}-h_{k}^{i j, n}\right)\right)-1\right)- \\
& \frac{1}{\theta} \sum_{i j}\left(q_{i j}^{n}+\alpha^{n} \cdot\left(v_{i j}^{n}-q_{i j}^{n}\right)\right) \times \\
& \left(\ln \left(q_{i j}^{n}+\alpha^{n} \cdot\left(v_{i j}^{n}-q_{i j}^{n}\right)\right)-1\right)- \\
& \sum_{i j} \int_{0}^{q_{i j}^{n}+\alpha^{n} \cdot\left(v_{i j}^{n}-q_{i j}^{n}\right)} D_{i j}^{-1}(x) d x
\end{aligned}
$$

subject to:

$$
0 \leq \alpha^{n} \leq 1 .
$$

Step 4. Updating flows:

$$
\begin{aligned}
& f_{a}^{n+1}=f_{a}^{n}+\alpha^{n} \cdot\left(y_{a}^{n}-f_{a}^{n}\right), \forall a ; \\
& q_{i j}^{n+1}=q_{i j}^{n}+\alpha^{n} \cdot\left(v_{i j}^{n}-q_{i j}^{n}\right), \forall i, j ; \\
& h_{k}^{i j, n+1}=h_{k}^{i j, n}+\alpha^{n} \cdot\left(g_{k}^{i j, n}-h_{k}^{i j, n}\right), \forall i, j, k .
\end{aligned}
$$

Step 5. Termination:

- Calculate the error $\varepsilon_{1}$ :

$$
\varepsilon_{1}=\frac{\sqrt{\sum_{i j} \sum_{k}\left(h_{k}^{i j, n+1}-h_{k}^{i j, n}\right)^{2}}}{\sum_{i j} \sum_{k} h_{k}^{i j, n}} .
$$

If $\varepsilon_{1}<\varepsilon_{0}$ (where $\varepsilon_{0}$ is the maximum permissible error), continue this step. Otherwise, increment iteration counter $n=n+1$ and go to Step 2.

- Calculate $t_{a}^{n+1}, t_{k}^{i j, n+1}, S_{i j}^{n+1}, D_{i j}\left(S_{i j}^{n+1}\right)$ and $P_{k}^{i j, n+1}$ by equations given above.

- Calculate the error $\varepsilon_{2}$ :

$$
\varepsilon_{2}=\frac{\sqrt{\sum_{i j} \sum_{k}\left(h_{k}^{i j, n+1}-D_{i j}\left(S_{i j}^{n+1}\right) \cdot P_{k}^{i j, n+1}\right)^{2}}}{\sum_{i j} \sum_{k} h_{k}^{i j, n+1}} .
$$

If $\varepsilon_{2}<\varepsilon_{0}^{\prime}$ (where $\varepsilon_{0}^{\prime}$ is the maximum permissible error), output the solution set $\left\{f_{a}^{n+1}\right\}$, $\left\{q_{i j}^{n+1}\right\}$ and $\left\{h_{k}^{i j, n+1}\right\}$, and stop the whole solving procedure. Otherwise, increment iteration counter $n=n+1$ and go to Step 2 . 


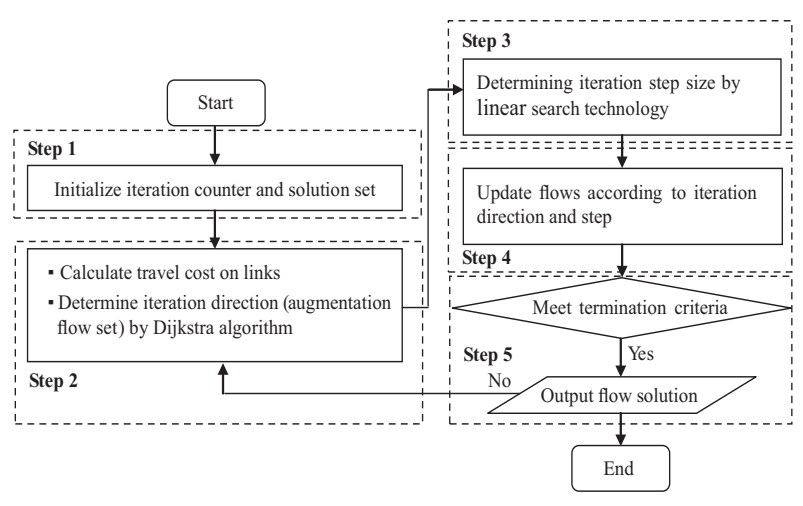

Fig. 2. Solution procedure for elastic-demand SUE model

Remark 6. The whole procedure is essentially a descent iteration process. In Step 2, Dijkstra's algorithm and the shortest-path assignment are used to determine iteration direction. In Step 3, Fibonacci method of linear search technology is used to determine iteration step size. Detailed descriptions of Dijkstra's algorithm and a Fibonacci method may refer to some literature (Wang 2004; Li 2006).

Remark 7. It can be proved in mathematics that updated flows in Step 4 in each iterative loop inevitably meet flow constraints of the program. In other words, this algorithm transforms constrained optimization problem into unconstrained optimization problem, so that feasible region need not be considered, which leads to the simplification in solving process. The proof is below.

Proof. In elastic-demand SUE model, the feasible region caused by flow constraints ( $1 \mathrm{~b}-\mathrm{d})$ is a convex set. Let $X_{1}$ and $X_{2}$ be $\left\{h_{k}^{i j, n}, f_{a}^{n}, q_{i j}^{n}\right\}$ and $\left\{g_{k}^{i j, n}, v_{a}^{n}, v_{i j}^{n}\right\}$, respectively, which are two points in this convex set. According to characteristics of convex set, the line segment connecting $X_{1}$ and $X_{2}$ is also in this convex set. Let $X_{3}$ be $\left\{h_{k}^{i j, n+1}, f_{a}^{n+1}, q_{i j}^{n+1}\right\}$, which is equal to $\left(1-\alpha^{n}\right) \cdot X_{1}+$ $\alpha^{n} \cdot X_{2}$. Then $X_{3}$ is in line segment $X_{1} X_{2}$ because value range of $\alpha^{n}$ is $[0,1]$. Thus, $X_{3}$ is in the convex set (feasible region) and the updated flows $\left\{h_{k}^{i j, n+1}, f_{a}^{n+1}, q_{i j}^{n+1}\right\}$ meet flow constraints.

Remark 8. In Step 5, there are two iteration termination criteria. The first criterion tests flow change between two adjacent iterations. If the change is small enough, it indicates that the objective function value can hardly be improved through the flow adjustment in certain iteration direction. In this case, the second criterion should be applied to judge whether current flow set is the final assignment result by an implicit function of flow solution. If current flow set meets the second criterion, it is the solution to implicit function (9). In this case, this flow set also serves as the solution to elastic-demand SUE model, which can be referred to as the global optimal solution because implicit functions (9) and (10) have equivalence relationship with elastic-demand SUE model. Thus, a flow set which meets the second, theo- retically speaking, the first criterion is not necessary. Despite this, the computational efficiency would descend sharply without the first criterion, because the computational complexity in Criterion 2 is much higher than that in Criterion 1. Moreover, for most cases, if the flow sets in iteration process can not meet Criterion 1, then they can not meet Criterion 2 consequentially.

Compared with previous algorithms for elastic-demand SUE model, this algorithm mainly has following advantages:

- the algorithm can obtain the final assignment results, rather than merely the implicit functions of flow solution;

- the algorithm is appropriate for any general traffic network, not confined to some specific ones such as public transit network;

- the algorithm produces no fictitious link in the whole solving procedure, which avoids the confusion caused by fictitious links and added links in the upper-level subprogram.

\subsection{Algorithm for Bi-Level Program}

Bi-level programs are generally difficult to solve by traditional calculus-based optimization methods, not only because evaluation of the upper-level objective function requires solving the lower-level subprogram, but the feasible solution set is too large as well. To solve the bi-level programming model proposed in this paper, a heuristic algorithm is developed, which consists of the proposed algorithm for elastic-demand SUE model and a modified GA. GA is the main body of the whole algorithm, which handles the nonlinear and non-convex nature of the bi-level program. Compared with other heuristic algorithms, GA has two main advantages:

- the operation object of GA is a group of feasible solutions rather than a single solution, which avoids trapping in local optimum to some extent;

- the objective function need not meet certain requirements such as differentiability.

Despite the above stated advantages, slow convergence rate and premature convergence exist in basic GA. Hence, some operators are designed to overcome these shortcomings, including elitist selection, adaptive cross, adaptive mutation and niche technology. Main steps of the solution procedure as depicted in Fig. 3 are summarized:

Step 1. Define GA parameters: mutation probability $p_{m 0}$, crossover probability $p_{c 0}$, group sizes $(N \& M, M<N)$, maximum number of generations $k_{\max }$. Initialize counter for the number of generations $k$ and a set of initial solutions (initial group $\{Y(0)\}=\left(Y_{1}(0), Y_{2}(0), \ldots, Y_{N}(0)\right)$. Step 2. Evaluate the objective function of every solution $Y_{i}(k)$ in group $\{Y(k)\}$ and calculate the fitness value of every solution.

Step 3. Select solutions with relatively high fitness value in group $\{Y(k)\}$ to produce a new group $\{X(k)\}$ of size $M$. 


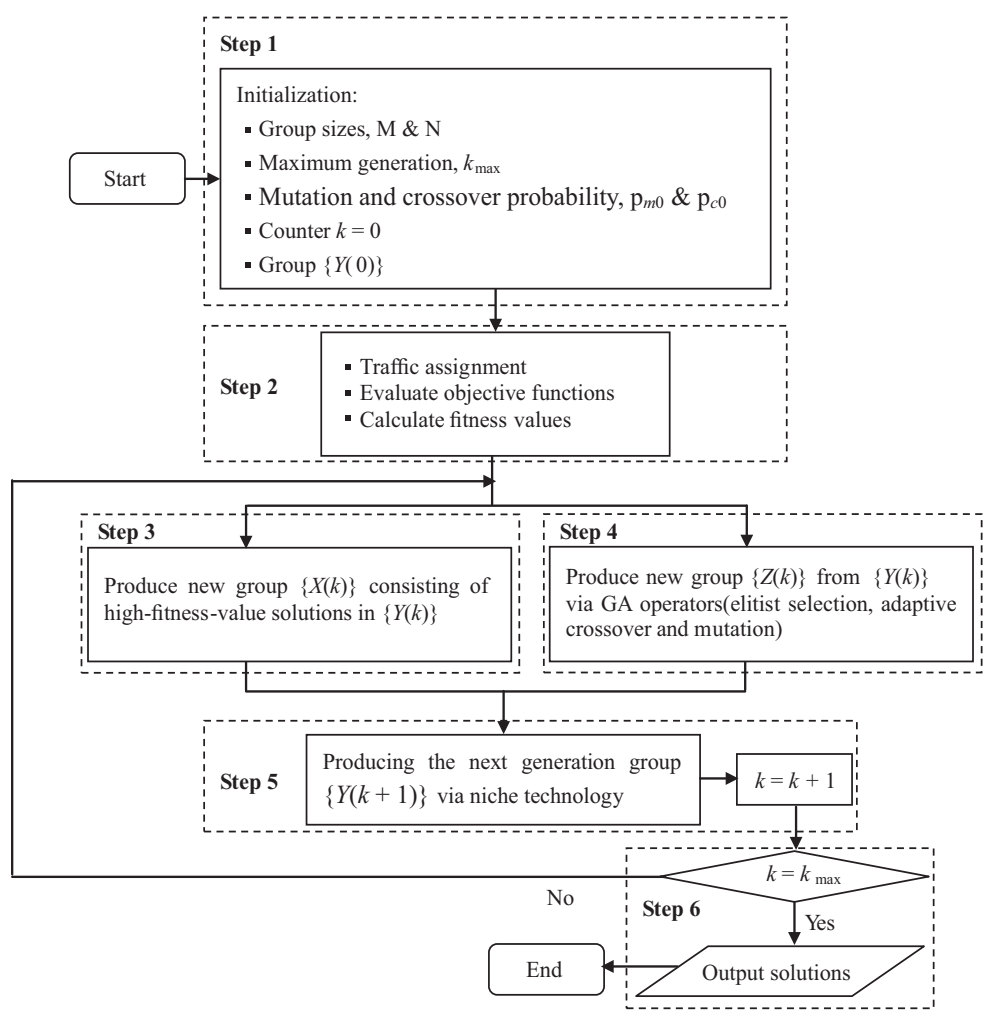

Fig. 3. Solution procedure for proposed bi-level program

Step 4. Evolve all solutions in group $\{Y(k)\}$ via GA operators: elitist selection, adaptive crossover, and adaptive mutation, resulting in producing another new group $\{Z(k)\}$ of size $N$. Calculate the fitness value of every solution $Z_{i}(k)$.

Step 5. Select $N$ solutions from group $\{X(k)\}$ and group $\{Z(k)\}$ with the help of niche technology, resulting in producing the next generation group $\{Y(k+1)\}$. Increment $k=k+1$.

Step 6. If $k=k_{\max }$, output the solution with the maximum fitness value in the last generation. Otherwise, go to Step 3.

Remark 9. In Step 2, evaluation of objective functions of every solution requires solving of the lowerlevel subprogram to obtain flow set $\left\{f_{a}\right\},\left\{q_{i j}\right\}$ and $\left\{h_{k}{ }^{i 1}\right\}$ by algorithm in Section 2.1. The fitness value of every solution is expressed as the difference between objective function value in the upper level of the reformed network and that of the original network. When a solution doesn't meet the saturation level constraint, the construction cost and off-gas emissions will be expanded several times to drop its fitness value, avoiding it being the final solution.

Remark 10. In Step 4, elitist selection, adaptive crossover and adaptive mutation operators are designed in order to improve convergence rate of GA. Elitist selection operator finds out the solution with maximum fitness value in a group which does not participate in crossover and mutation. Adaptive crossover and mutation operators determine crossover and mutation probabilities respectively according to fitness value. If fitness value of a solution is higher than average one in a group, its crossover and mutation probabilities will descend. Calculation method is shown below:

$$
\begin{aligned}
& p_{c}=\left\{\begin{array}{l}
p_{c 0}-p_{c 0} \cdot \frac{f^{\prime}-f_{\text {ave }}}{f_{\max }-f_{\text {ave }}}, \text { when } f^{\prime} \geq f_{\text {ave }} ; \\
p_{c 0}, \text { when } f^{\prime}<f_{\text {ave }} ;
\end{array}\right. \\
& p_{m}=\left\{\begin{array}{l}
p_{m 0}-p_{m 0} \cdot \frac{f-f_{\text {ave }}}{f_{\max }-f_{\text {ave }}}, \text { when } f \geq f_{\text {ave }} ; \\
p_{m 0}, \text { when } f<f_{\text {ave }}
\end{array}\right.
\end{aligned}
$$

where: $p_{c}$ and $p_{m}$ are adaptive crossover and mutation probabilities respectively; $p_{c 0}$ and $p_{m 0}$ are ordinary crossover and mutation probabilities respectively; $f$ is fitness value of a solution; $f^{\prime}$ is the higher value in two solutions which make crossover operation; $f_{\max }$ and $f_{\text {ave }}$ are the maximum and average fitness values in a group respectively. Elitist selection, adaptive crossover and adaptive mutation operators carry out the persistence of solutions with relatively high values, which avoid these solutions trapping in circulation between appearance and disappearance and result in an improvement in convergence rate consequently.

Remark 11. In Step 5, niche technology is designed to overcome premature convergence in basic GA, because it can guarantee diversity of solutions in every generation so as to avoid all solutions approaching some local optimum simultaneously. In the niche technology, difference between each two solutions of two groups $\{X(k)\}$ and $\{Z(k)\}$ - are calculated as follows: 


$$
\left\|Y_{i}-Y_{j}\right\|=\sum_{k=1}^{n}\left|y_{i k}-y_{j k}\right|,
$$

where: $Y_{i}$ is a design vector (solution) and $y_{i k}$ is a design variable of $Y_{i} ; i=1 \sim(M+N-1), j=(i+1) \sim(M+N)$. If the difference is less than a certain value $r$, these two solutions are regarded as similar solutions. The solution with lower fitness value will be punished by cutting its fitness value in half.

Detailed description of GA solution procedure may refer to related literature (Xu et al. 2009).

\section{Numerical Example}

In this section, the proposed bi-level programming model for RNDP and its algorithm are applied to a specific road network, in order to demonstrate their validation.

\subsection{Problem Description}

Fig. 4 shows an original road network, link attributes and travel demands in the network as well as maximum potential travel demands. Necessary to say, any two nodes can be connected to form a probably added link. However, if a large number of probably added links are taken into consideration, the calculation amount will be enlarged remarkably. Therefore, only a few probably added links are chosen based on engineering experience in practice (four in this example).

The task is to provide a road network design scheme (improving existing links and adding new links), aiming to maximize the whole social benefit.

\subsection{Problem Solving}

Two main stages are taken to solve the problem:

Stage I: Model (15) is established and the following parameters are used:

1) Parameters in upper level:

According to reference (Wang 2002), the parameters in the upper level are set as follows:

- Unit-matching coefficients: $\theta_{1}=0.2, \theta_{2}=0.03$ (under the circumstances that the unit of travel demand is $p c u / h$, the unit of construction cost is 10000 yuan and the unit of off-gas emissions is $g / h$ );

- Upper and lower bounds of saturation level: $R_{1}=0.25, R_{h}=0.95$;

- Coefficients in off-gas emissions calculation: $b_{0}=167.154, b_{1}=-5.291, b_{2}=0.0662$, $b_{3}=-0.0003$ (The unit of speed is $\mathrm{km} / \mathrm{h}$ and the unit of off-gas emissions coefficient is $\mathrm{g} / \mathrm{pcu} / \mathrm{km})$.

2) Parameters in lower level:

$-\theta=2.0, \beta=0.05$ (gained by the method presented in Section 1.1).

3) Parameters in GA:

According to the reference (Han 2010), the parameters in GA are set as follows:

- Group size: $N=50, M=25$;

- Maximum number of generations $=150$;

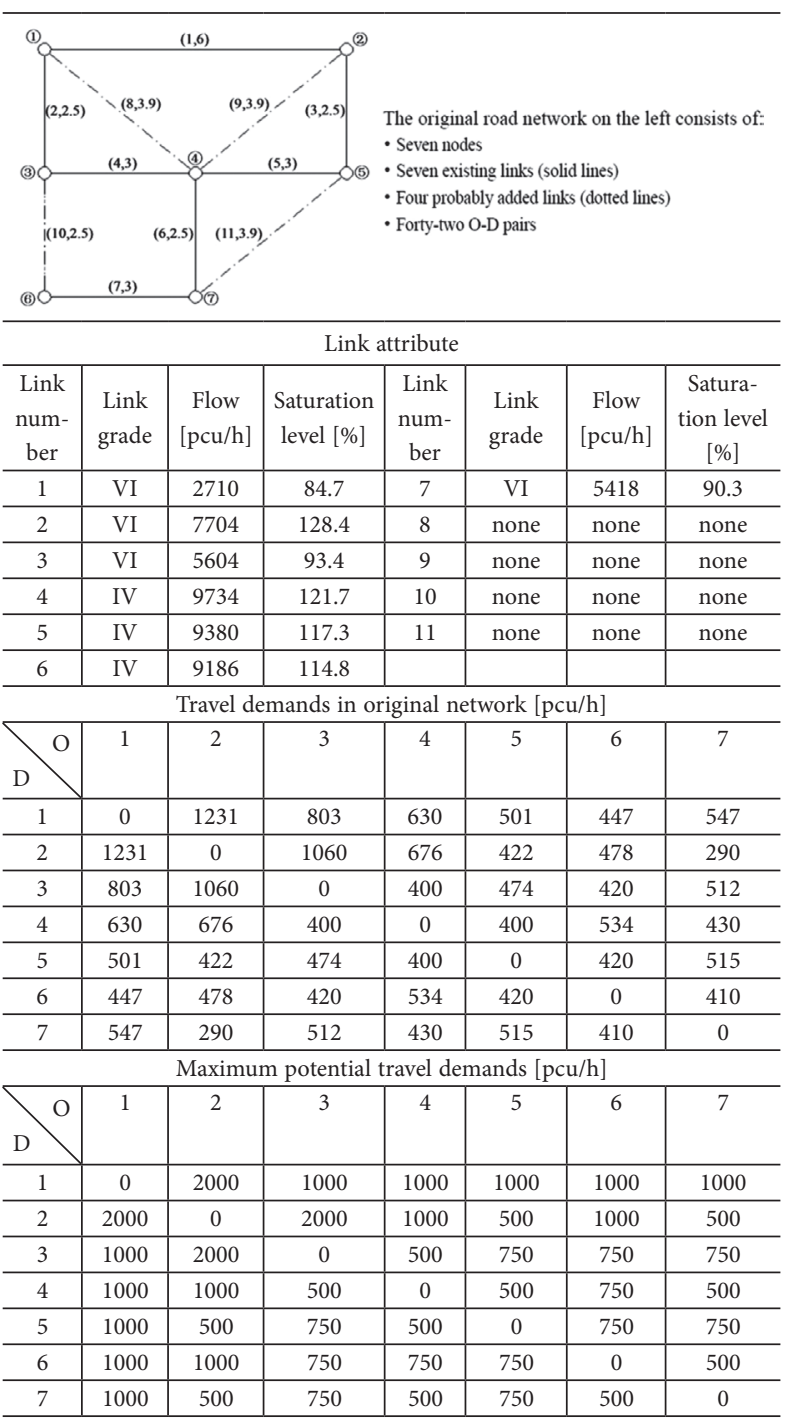

Fig. 4. Original network and travel demand

- Ordinary crossover and mutation probabilities: $p_{c 0}=0.5, p_{m 0}=0.02$;

- In niche technology, $r=3$.

Stage II: As it is said in Introduction, during the last decade, some advanced researches have concluded that GA has advantages over other heuristic optimization techniques in finding optimal solutions of RNDP. In order to make preparation and demonstrate the effectiveness of the modified GA proposed in this paper, both proposed GA and the basic GA are applied to solve the established bi-level programming model in this example. Using the MATLAB 7.0 software and its GA toolbox (Huang et al. 2005), programs are developed to solve the problem, through which the road network design schemes are obtained.

\subsection{Results and Analysis}

The programs are implemented on a personal computer with Core V 3.2GHz, 2.0GB. Initial points for the optimization are produced automatically by the MATLAB 7.0 software (http://se.mathworks.com). Results are shown as follows. 
Table 2. Results of two GAs

\begin{tabular}{ccccc}
\hline Algorithm & Generations & CPU time $[\mathrm{s}]$ & Objective function value $[\mathrm{pcu} / \mathrm{h}]$ & Fitness value $[\mathrm{pcu} / \mathrm{h}]$ \\
\hline Basic GA & 111 & 327 & 6327 & 1563 \\
\hline Proposed GA & 78 & 212 & 6969 & 2205 \\
\hline
\end{tabular}

Table 2 shows that when reaching optimum, basic GA needs more generations than the modified GA. Moreover, the modified GA proposed in this paper obtains a road network design scheme with higher social benefit, since it overcomes the drawbacks of slow convergence rate and premature convergence in the basic GA. In terms of social benefit, the fitness value of the final solution from the modified GA is 2205, which indicates that the whole social benefit of reformed network is higher than that of original one and the difference is equivalent to such a benefit that can meet travel demand of 2205 passenger car units per hour in the network. The specific design scheme and situation of reformed network are shown in Table 3.

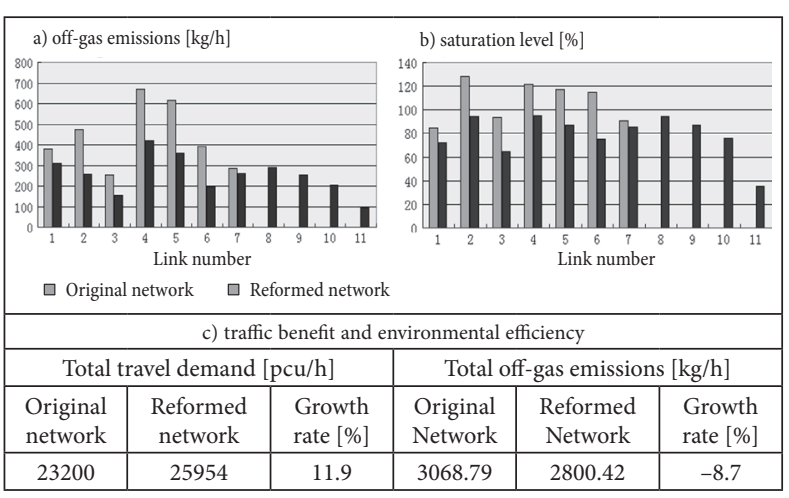

Fig. 5. Comparison between original and reformed networks
In the designing scheme, 109800000 yuan is spent in reforming the network. Comparisons between original and reformed networks are shown in Fig. 5.

According to Fig. 5, it can be found that the average off-gas emissions per car in original and reformed networks are $0.132 \mathrm{~kg} / \mathrm{h}$ and $0.108 \mathrm{~kg} / \mathrm{h}$ respectively. It can be concluded that the design scheme in Table 3 not only brings about higher traffic benefit and environmental efficiency, but also reduces the saturation level of the road network markedly. As a result, the model and algorithm proposed in this paper are effective and reasonable, which shows bright prospects in the application for solving RNDP.

\section{Conclusions}

In this paper, RNDP has been studied. A bi-level programming model and its algorithm have been proposed and applied to a specific numerical example. Main achievements and innovations are listed as follows:

\section{Models:}

- The lower level subprogram adopts elastic-demand SUE model to coincide well with uncertainties in users' behavior. In order to guarantee accuracy of flow prediction, parameter calibration method for the model is designed to determine parameter values quantitatively, not empirically as previous methods.

Table 3. Link attributes and travel demand in reformed network

\begin{tabular}{|c|c|c|c|c|c|c|c|}
\hline \multicolumn{8}{|c|}{ Link attribute } \\
\hline $\begin{array}{c}\text { Link } \\
\text { number }\end{array}$ & $\begin{array}{l}\text { Link } \\
\text { grade }\end{array}$ & $\begin{array}{c}\text { Flow } \\
{[\mathrm{pcu} / \mathrm{h}]}\end{array}$ & $\begin{array}{c}\text { Saturation } \\
\text { level [\%] }\end{array}$ & Link number & Link grade & $\begin{array}{c}\text { Flow } \\
{[\mathrm{pcu} / \mathrm{h}]}\end{array}$ & $\begin{array}{c}\text { Saturation } \\
\text { level [\%] }\end{array}$ \\
\hline 1 & VI & 2312 & 72.3 & 7 & IV & 5114 & 85.2 \\
\hline 2 & IV & 5676 & 94.6 & 8 & VI & 3026 & 94.6 \\
\hline 3 & IV & 3872 & 64.5 & 9 & VI & 2772 & 86.6 \\
\hline 4 & III & 7660 & 94.8 & 10 & $\mathrm{~V}$ & 3638 & 75.8 \\
\hline 5 & III & 6956 & 87.0 & 11 & VI & 1134 & 35.4 \\
\hline 6 & III & 5982 & 74.8 & & & & \\
\hline \multicolumn{8}{|c|}{ Travel demands $[\mathrm{pcu} / \mathrm{h}]$} \\
\hline & 1 & 2 & 3 & 4 & 5 & 6 & 7 \\
\hline 1 & 0 & 1252 & 845 & 823 & 595 & 694 & 638 \\
\hline 2 & 1252 & 0 & 1197 & 733 & 429 & 539 & 327 \\
\hline 3 & 845 & 1197 & 0 & 408 & 504 & 616 & 541 \\
\hline 4 & 823 & 733 & 408 & 0 & 411 & 545 & 441 \\
\hline 5 & 595 & 429 & 504 & 411 & 0 & 464 & 563 \\
\hline 6 & 694 & 539 & 616 & 545 & 464 & 0 & 412 \\
\hline 7 & 638 & 327 & 541 & 441 & 563 & 412 & 0 \\
\hline
\end{tabular}


- The upper level subprogram establishes a nonlinear integer programming model with the goal of maximizing consumer surplus. It takes multi-factors into consideration, including travel demand, construction cost, off-gas emissions and saturation level. Moreover, the upper level model can represent different importance of traffic benefit, economical efficiency and environmental efficiency through adjusting unit-matching coefficients in construction projects.

2. Algorithms:

- A utility algorithm for elastic-demand SUE model is developed based on descent iteration method, Dijkstra's algorithm and linear search technology. The new algorithm can resolve some limitations in the previous algorithms such as the restrictions in application range and the production of fictitious links.

- A modified GA for the established RNDP model is developed, in which elitist selection operator, adaptive cross operator, adaptive mutation operator and niche technology are designed to overcome the drawbacks of slow convergence rate and premature convergence in basic GA.

3. Example:

- A numerical example is conducted. With the application of the proposed bi-level model and related algorithms, a design scheme with relatively higher social benefits is gained through less calculation. Results demonstrate that the proposed model and algorithms are effective and reasonable.

It should be mentioned that only two measures (adding new links and improving existing links) have been taken into consideration in RNDP so far. Future researches will also consider other measures such as reversible lanes and the congestion charge. On the computational side, method of setting reasonable maximum number of generations should be further explored to avoid unnecessary operation in future researches.

\section{Acknowledgements}

This research was supported by the National High Technology Research and Development Program of China (863 Program, Grant No 2012AA112304).

\section{References}

Anderson, W. P.; Kanaroglou, P. S.; Miller, E. J.; Buliung, R. N. 1996. Simulating automobile emissions in an integrated urban model, Transportation Research Record 1520: 71-80. http://dx.doi.org/10.3141/1520-09

Asakura, Y.; Sasaki, T. 1990. Formulation and feasibility test of optimal road network design model with endogenously determined travel demand, in Transport Policy, Management \& Technology Towards: Selected Proceedings of the Fifth World Conference on Transport Research, July 1989, Yokohama, Japan, 351-365.

Bagloee, S. A.; Ceder, A. 2011. Transit-network design methodology for actual-size road networks, Transportation Research Part B: Methodological 45(10): 1787-1804.

http://dx.doi.org/10.1016/j.trb.2011.07.005
Bergum, J.; Cong, B.; Sharma, S. 1992. Simulation of tree structures on Fibonacci cubes, in Proceedings of the First International Conference on Computer Communications and Networks, 8-10 June 1992, San Diego, California, USA, 279-283.

Braess, D. 1968. Über ein Paradoxon aus der Verkehrsplanung, Unternehmensforschung 12: 258-268. (in German).

Cantarella, G. E.; Vitetta, A. 2006. The multi-criteria road network design problem in an urban area, Transportation 33(6): 567-588. http://dx.doi.org/10.1007/s11116-006-7908-z

Cascetta, E. 2001. Transportation Systems Engineering: Theory and Methods. Springer-Verlag US. 710 p. http://dx.doi.org/10.1007/978-1-4757-6873-2

Chen, A.; Kim, J.; Lee, S.; Kim, Y. 2010. Stochastic multi-objective models for network design problem, Expert Systems with Applications 37(2): 1608-1619. http://dx.doi.org/10.1016/j.eswa.2009.06.048

Chen, A.; Zhou, Z.; Chootinan, P.; Ryu, S.; Yang, C.; Wong, S. C. 2011. Transport network design problem under uncertainty: a review and new developments, Transport Reviews 31(6): 743-768. http://dx.doi.org/10.1080/01441647.2011.589539

Chen, M.; Alfa, A. S. 1991. Algorithms for solving fisk's stochastic traffic assignment model, Transportation Research Part B: Methodological 25(6): 405-412. http://dx.doi.org/10.1016/0191-2615(91)90033-F

Chiou, S.-W. 2009. A subgradient optimization model for continuous road network design problem, Applied Mathematical Modelling 33(3): 1386-1396.

http://dx.doi.org/10.1016/j.apm.2008.01.020

Drezner Z.; Salhi, S. 2002. Using hybrid metaheuristics for the one-way and two-way network design problem, Naval Research Logistics 49(5): 449-463.

http://dx.doi.org/10.1002/nav.10026

Drezner, Z.; Wesolowsky, G. O. 2003. Network design: selection and design of links and facility location, Transportation Research Part A: Policy and Practice 37(3): 241-256. http://dx.doi.org/10.1016/S0965-8564(02)00014-9

Farvaresh, H.; Sepehri, M. M. 2011. A single-level mixed integer linear formulation for a bi-level discrete network design problem, Transportation Research Part E: Logistics and Transportation Review 47(5): 623-640. http://dx.doi.org/10.1016/j.tre.2011.02.001

Florian, M. 1977. An improved linear approximation algorithm for the network equilibrium (packet switching) problem, Proceedings of the 1977 IEEE Conference on Decision and Control Including the 16th Symposium on Adaptive Processes and A Special Symposium on Fuzzy Set Theory and Applications, 7-9 December 1977, New Orleans, LA, USA, 812-818. http://dx.doi.org/10.1109/CDC.1977.271681

Friesz, T. L.; Anandalingam, G.; Mehta, N. J.; Nam, K.; Shah, S. J.; Tobin, R. L. 1993. The multiobjective equilibrium network design problem revisited: a simulated annealing approach, European Journal of Operational Research 65(1): 44-57. http://dx.doi.org/10.1016/0377-2217(93)90143-B

Friesz, T. L.; Cho, H.-J.; Mehta, N. J.; Tobin, R. L.; Anandalingam, G. 1992. A simulated annealing approach to the network design problem with variational inequality constraints, Transportation Science 26(1): 18-26. http://dx.doi.org/10.1287/trsc.26.1.18

Guo, X. 2009. Case Analysis in Traffic Engineering. Beijing, China: China Railway Publishing House. 464 p. (in Chinese). 
Han, R. 2010. Genetic Algorithm Principle and Application Example. Beijing, China: Arms Industry Press. 443 p. (in Chinese).

He, Y.; Yang, S.; Xu, Q. 2013. Short-term cascaded hydroelectric system scheduling based on chaotic particle swarm optimization using improved logistic map, Communications in Nonlinear Science and Numerical Simulation 18(7): 1746-1756. http://dx.doi.org/10.1016/j.cnsns.2012.11.003

Huang, Y.; Ma, L.; Liu, H. 2005. Modelling Simulation Exploration and Advanced Engineering Application Based on Matlab 7.0 and Simulink 6.0. Beijing, China: Tsinghua University Press. 354 p. (in Chinese).

Karoonsoontawong, A.; Waller, S. T. 2006. Dynamic continuous network design problem: linear bilevel programming and metaheuristic approaches, Transportation Research Record 1964: 104-117. http://dx.doi.org/10.3141/1964-12

Kuang, A.-W.; Wang, Z.-W.; Li, B.-L. 2007. Model and its solution for stochastic user equilibrium traffic assignment with multiple user classes and variable demand, Journal of Changsha University of Science and Technology (Natural Science) (2): 16-22. (in Chinese).

Kung, R.-M.; Hanson, E.; Ioannidis, Y.; Sellis, T.; Shapiro, L.; Stonebraker, M. 1984. Heuristic search in database systems, in Proceedings of the 1st International Workshop on Expert Database Systems, 24-27 October 1984, Kiawah Island, SC, 1: 96-107.

Li, Y. 2006. Optimization Design in Engineering. Beijing, China: Tsinghua University Press. 179 p. (in Chinese).

Li, Z.-C.; Gu, Q.; Shi, F. 2001. Toll model and algorithm of road jammed with traffic based on elastic demand, Journal of Traffic and Transportation Engineering 1(3): 81-85. (in Chinese).

Luathep, P.; Sumalee, A.; Lam, W. H. K.; Li, Z.-C.; Lo, H. K. 2011. Global optimization method for mixed transportation network design problem: a mixed-integer linear programming approach, Transportation Research Part B: Methodological 45(5): 808-827. http://dx.doi.org/10.1016/j.trb.2011.02.002

Ministry of Housing and Urban-Rural Development of People's Republic of China. 1991. Design Specifications of Urban Roads. Beijing, China: China Construction Industry Press. 58 p. (in Chinese).

Ortega, J. M.; Rheinboldt, W. C. 1970. Iterative Solution of Nonlinear Equations in Several Variables. Academic Press. $572 \mathrm{p}$.

Patriksson, M. 2008. On the applicability and solution of bilevel optimization models in transportation science: a study on the existence, stability and computation of optimal solutions to stochastic mathematical programs with equilibrium constraints, Transportation Research Part B: Methodological 42(10): 843-860. http://dx.doi.org/10.1016/j.trb.2008.05.001

Poorzahedy, H.; Abulghasemi, F. 2005. Application of Ant System to network design problem, Transportation 32(3): 251-273. http://dx.doi.org/10.1007/s11116-004-8246-7

Poorzahedy, H.; Rouhani, O. M. 2007. Hybrid meta-heuristic algorithms for solving network design problem, European Journal of Operational Research 182(2): 578-596. http://dx.doi.org/10.1016/j.ejor.2006.07.038

Qin, J.; Ni, L.-L.; Dong, L.-Y.; Shi, F.; Miao, L.-X. 2010. Bi-level programming model and algorithm for transportation network design problem considering sustainable development, Journal of Transportation Systems Engineering and Information Technology 10(4): 111-117. (in Chinese).
Sharma, S.; Ukkusuri, S. V.; Mathew, T. W. 2009. Pareto optimal multiobjective optimization for robust transportation network design problem, Transportation Research Record 2090: 95-104. http://dx.doi.org/10.3141/2090-11

Highway Capacity Manual. 2010. Transportation Research Board. 5th edition. $1650 \mathrm{p}$.

Wang, D. Z. W.; Lo, H. K. 2010. Global optimum of the linearized network design problem with equilibrium flows, Transportation Research Part B: Methodological 44(4): 482-492. http://dx.doi.org/10.1016/j.trb.2009.10.003

Wang, W. 2004. System Analysis Method of Road Traffic System. Beijing, China: People Communication Press. 243 p. (in Chinese).

Wang, W.; Xiang, Q.; Chang, Y. 2002. Analysis Method of Energy Consumption and Environmental Effect in Urban Transportation System. Beijing, China: Science Press. 420 p. (in Chinese).

Xiong, Y.; Schneider, J. 1995. Processing of Constraints in Transportation Network Design Problem, Journal of Computing in Civil Engineering 9(1): 21-28. http://dx.doi.org/10.1061/(ASCE)0887-3801(1995)9:1(21)

Xu, T.; Wei, H.; Hu, G. 2009. Study on continuous network design problem using simulated annealing and genetic algorithm, Expert Systems with Applications 36(2): 1322-1328. http://dx.doi.org/10.1016/j.eswa.2007.11.023

Yang, H.; Bell, M. G. H. 1998. Models and algorithms for road network design: a review and some new developments, Transport Reviews 18(3): 257-278. http://dx.doi.org/10.1080/01441649808717016

Zhao, T.; Guo, T.; Gao, Z. 2005. A optimal model and solution algorithm for maximal OD travel demand in urban transport discrete network design problem under environment objective, China Civil Engineering Journal 38(3): 119-124. (in Chinese).

Zhou, Z. 2008. Advanced Theory Method and Model for Urban Transportation Planning. Beijing, China: China Railway Publishing House. 237 p. (in Chinese).

Zhou, J. 2001. Analysis and Optimization of Urban Transportation System. Nanjing, China: Southeast University Press. 259 p. (in Chinese).

Zhou, J.; Xu, Y. 2001. Stochastic user equilibrium assignment with elastic demand and its application, Journal of Systems Engineering (2): 88-94. (in Chinese). 